\title{
Variations
}

Variations

Revue internationale de théorie critique

$9 / 10 \mid 2007$

Les frontières de la politique

\section{La fin de la nuit}

Les trotskistes et l'« entrisme » au Parti Communiste Français

\section{Denis Berger}

\section{(2) OpenEdition}

Journals

Édition électronique

URL : http://journals.openedition.org/variations/471

DOI : 10.4000/variations.471

ISSN : 1968-3960

Éditeur

Les amis de Variations

Édition imprimée

Date de publication : 1 juin 2007

Pagination : 67-78

Référence électronique

Denis Berger, «La fin de la nuit », Variations [En ligne], 9/10 | 2007, mis en ligne le 01 décembre 2012,

consulté le 02 mai 2019. URL : http://journals.openedition.org/variations/471 ; DOI : 10.4000/

variations.471

Ce document a été généré automatiquement le 2 mai 2019.

Les ami•e•s de Variations 


\title{
La fin de la nuit
}

\author{
Les trotskistes et l'« entrisme » au Parti Communiste Français
}

\section{Denis Berger}

1 Débutons ce témoignage par quelques renseignements d'ordre personnel. Ils permettront de situer mon point de vue et mes remarques.

2 J'ai adhéré au Parti Communiste Internationaliste (section française de la IVe Internationale) en mars 1950, après quelques mois d'activité au titre de sympathisant. J'avais un peu moins de 18 ans, et jusque-là, je m'étais situé dans l'orbite du mouvement anarchiste. Le choix que je fis du trotskisme fut influencé par une série de lectures ( $L a$ Révolution Trahie, en particulier) et de discussions avec des militants du PCI. Ma décision fut influencée, outre la fascination exercée par le personnage de Trotsky, par les positions stratégiques de la IVe Internationale: à une critique impitoyable du stalinisme, particulièrement nécessaire en ces années de domination bureaucratique, s'ajoutait logiquement une fidélité quasi absolue à la révolution d'Octobre, ses tactiques et ses principes. À partir de cette base, on pouvait être communiste critique sans renoncer au réalisme qui commandait de prendre en compte l'influence de masse qu'exerçaient nombre d'organisations staliniennes ${ }^{1}$. La IVe Internationale avait survécu à la guerre, en dépit des conditions extrêmement difficiles de sa participation aux luttes, compte tenu de la faiblesse de ses effectifs et, plus encore peut-être, des campagnes meurtrières menées pas les staliniens. Ceux qui rejoignaient le mouvement trotskiste en cette période ressentaient fortement la vigueur militante des groupes constitutifs de l'Internationale. Toutefois, courage et combativité ne suffisent pas à déterminer une orientation politique. Or, la IVe Internationale vivait, sans le reconnaitre officiellement, sur un échec politique : née en 1938, sur la lancée d'un travail amorcé dès le début de la décennie, l'organisation, à la suite de Léon Trotsky, avait adopté une perspective de succès de masse à court ou moyen terme. Dans une large mesure, elle fondait sa démarche stratégique sur l'hypothèse d'un renouvellement de l'expérience léniniste au cours de la première guerre mondiale : le conflit politico-militaire verrait un effondrement du régime bureaucratique en Union Soviétique et, à l'échelle mondiale, les partis communistes, privés de 
l'orientation fournie par Moscou, se rallieraient à leur bourgeoisie nationale, rejoignant ainsi la sociale-démocratie dans la trahison.

3 Ainsi s'ouvrirait un moment historique où les révolutionnaires auraient la possibilité de se battre pour le contrôle des masses révolutionnaires. Il est inutile de souligner longuement le caractère globalement erroné de pareille analyse ${ }^{2}$. Je n'ai fait référence aux impasses politiques dans lesquelles piétinait la IVe Internationale que pour décrire le cadre dans lequel se déroulaient les débats des trotskistes. Cadre dont les parties souterraines, cachées dans les débats officiels, occupaient néanmoins les positions clés. C'est ainsi que nombre de jeunes militants, comme moi désireux de suivre les débats, mais encore peu armés pour y participer activement, cherchaient en vain dans les écrits du « vieux » et de ses disciples des réponses aux questions de fond qu'ils se posaient et qui, par-delà l'actualité, envisageaient certains fondements du "bolchevisme", référence obligée des trotskistes. Le parti, les formes de la révolution, la "nature » de l'Etat soviétique, etc. autant de questions angoissantes qui, parce que non traitées (ou mal traitées) dans les confrontations de la IVe Internationale, pesaient sur la réflexion de chaque militant sur le mode de l'interrogation refoulée parce qu'angoissante.

Pourtant, la situation internationale n'apparaissait pas bouchée. La rupture entre la Yougoslavie et le « camp socialiste » constitué autour de l'URSS était en fait une crise sans précédent du système bureaucratique. Elle ouvrait, pour un avenir relativement rapproché, la perspective d'une remise en cause globale du stalinisme ${ }^{3}$. Par ailleurs, les anciens empires coloniaux - celui de la France en particulier - étaient le théâtre de luttes de libération nationale aux conséquences importantes. Bref, les possibilités d'actions révolutionnaires existaient et rendaient indispensable une réflexion approfondie sur la stratégie. La IVe Internationale mena ce débat, à l'occasion de son troisième congrès mondial. On ne peut dire de bonne foi que, malgré la qualité d'une partie des discussions, le résultat fut satisfaisant. En tout état de cause, une scission en fut la conclusion durable.

C'est autour des thèses présentées par Michel Pablo, principal animateur du Secrétariat International, que s'organisa la confrontation. Il ne m'est pas nécessaire de développer ici des considérations générales sur ce débat. Je voudrais simplement indiquer comment je l'ai vécu, en compagnie de quelques jeunes militants, étudiants pour la plupart. Nous avions une conscience obscure des failles des conceptions théoriques de l'Internationale, tout en ayant profondément intégré le schéma organisationnel centralisateur qui tendait à faire de la fédération de groupes minoritaires qu'était le mouvement trotskiste, une avant-garde fonctionnant comme "parti mondial de la révolution», sur le modèle de la Troisième Internationale à ses débuts.

6 Telles sont les raisons principales pour lesquelles je choisis, dans le débat violent qui se déroula dans la section française, le « camp » de Pablo, Ernest Germain et Pierre Frank. Le respect de la « direction internationale » m'apparaissait comme une démarche politique. Par ailleurs, et de façon beaucoup plus décisive, j'approuvais la démarche qui aboutissait à l'« entrisme sui generis ». Ladite démarche équivalait à une reconnaissance tacite de l'influence que conservaient les grandes organisations implantées dans le mouvement ouvrier, quelle que soit leur orientation politique. Le constat pouvait être fait là où la sociale-démocratie l'emportait (en Allemagne de l'Ouest ou en Grande-Bretagne par exemple), mais aussi dans les pays où le Parti communiste, fût-il aussi profondément stalinien qu'en France, avait su conquérir une base de masse. Pour combattre les directions bureaucratiques, les révolutionnaires devaient entreprendre un travail de relativement longue haleine à l'intérieur des partis en question : il s'agissait d'entraîner 
un maximum de militants hors du cercle de l'influence organisationnelle des bureaucrates en tout genre. On dégageait ainsi une perspective concrète de construction de partis révolutionnaires. Malheureusement, celle élaboration ne se réalisa pas dans une totale clarté. Les tenants de la ligne « entriste » ne se bornèrent pas à analyser les grands axes d'évolution des rapports de force internationaux dans le dessein de déterminer, autant que faire se pouvait, les possibilités immédiates et les formes concrètes de l'orientation proposée. Ils cédèrent au contraire à la tradition douteuse d'un certain marxisme, au demeurant fortement édulcoré par rapport aux aspects plus valables de la méthode marxienne ${ }^{4}$. À l'instar de dirigeants socialistes ou communistes, ils transformèrent les hypothèses que leur inspirait la situation mondiale en certitudes qui justifiaient la stratégie par eux proposée. C'est ainsi que s'imposa dans la discussion une vision des années à venir marquée par un déterminisme aussi absolu qu'indémontré. La troisième guerre mondiale allait éclater dans un délai de deux à cinq ans (nous étions au début des années cinquante). Elle aurait un caractère de classe, dans la mesure où elle serait l'affrontement entre le «camp» impérialiste, dirigé par les Etats-Unis et le " camp ", objectivement révolutionnaire, mené par les États "ouvriers", à vrai dire fortement dégénérés ou déformés. Dans ce contexte sulfureux, les grandes organisations $\mathrm{du}$ mouvement ouvrier seraient contraintes de faire un choix existentiel : certaines, d'origine communiste principalement, pourraient éventuellement «esquisser une orientation révolutionnaire »; les autres seraient en porte-à-faux par rapport aux réactions de la classe de la majorité des travailleurs; des "pans entiers » de leur masse d'adhérents se détacheraient sur la gauche.

7 Il y aurait beaucoup à dire encore sur ce débat fondamentalement confus à force de dogmatisme. Notons seulement une fois encore que l'idée importante selon laquelle la réalisation d'une perspective révolutionnaire mondiale, exigeait un travail de longue haleine en direction des organisations implantées, ne fut pas discutée avec précision. Quelle tactique suivre, autour de quels thèmes politiques adaptés au contexte ? Comment maintenir une expression autonome des révolutionnaires? Autant de problèmes dont la solution était attribuée au mouvement général des affrontements de classe. Le débat confus qui en résulta ne put contribuer à convaincre nombre de militants soucieux de maintenir une expression révolutionnaire critique qu'ils craignaient de voir disparaître avec l'entrisme. Quant aux jeunes gens dont j'étais, il leur restait à mettre en pratique « l'entrisme » et à élaborer une politique hors schéma. En attendant, survint la scission ${ }^{5}$.

\section{Militer au Parti Communiste Français ?}

8 En 1952, le PCI connaît donc une nouvelle scission. Les partisans de la stratégie « entriste $»^{6}$ défendue par le Secrétariat International sont, dans la section française, nettement minoritaires (ils doivent sans doute être une cinquantaine). Les cadres dotés d'une expérience militante sont peu nombreux et, en tout état de cause, sont publiquement connus comme trotskistes et ne peuvent en conséquence militer dans le mouvement communiste «officiel » qui à l'époque traverse une phase ultra stalinienne. L'intervention à l'intérieur du PCF échoit donc à de (très) jeunes militants. On peut deviner la difficulté qu'entraîne leur manque d'expérience. Mais, par ailleurs, peut-être sont-ils plus ouverts à la complexité d'une organisation comme le PCF.

9 Le parti de Maurice Thorez ne peut en effet faire l'objet d'une présentation simpliste, tant ses caractéristiques politiques et organisationnelles sont diverses, voire contradictoires. 
Le mouvement, né lors du congrès de Tours, en 1920, a connu des moments difficiles, notamment entre 1928 et 1934. Mais depuis le Front Populaire, les communistes constituent le premier parti politique français, tant du point de vue de l'influence électorale que du nombre de militants. Pareille situation a des conséquences évidentes sur les rapports que le parti entretient avec la masse de la population, qu'elle soit favorable ou non à la politique des communistes français : une organisation dotée d'un groupe parlementaire d'environ 183 députés et profondément intégrée dans le système républicain.

10 Pour cette raison, le PCF, en dépit de l'isolement qu'implique sa dépendance à l'univers stalinien, subit l'influence de la tradition politique française telle que l'expriment les institutions étatiques. L'organisation est profondément bureaucratique dans ses structures et son fonctionnement. Et elle doit cette peu glorieuse caractéristique au « modèle bolchevik» tel qu'il a dégénéré au fil de l'histoire de l'Union Soviétique. Mais le «stalinisme à la française » dont parlent rétrospectivement certains analystes (tel Roland Leroy) doit une part de sa spécificité aux facteurs nationaux que représentent les institutions républicaines. Dans le parti, la rigidité terrifiante qui étouffe tous les débats et qui provoque une sélection des cadres en fonction de leur docilité (au moins apparente) est due à Staline. Mais elle a également une origine nationale et répond aux critères d'étouffement de la démocratie qu'ont pu formuler, en d'autres temps et d'autres lieux, Robert Michels et tous ceux qui ont mené une recherche sur les "partis ouvriers » de l'Europe capitaliste. Les militants du PCF se trouvent par conséquent, dans leurs références idéologiques et leurs modalités d'actions quotidiennes, en proie à une ambiguïté fondamentale. Défenseurs inconditionnels de l'URSS, ils en reproduisent toutes les tares. Mais, en même temps, leur croyance en la réalité du communisme soviétique, malgré ses aspects lamentables, contribue à maintenir, pour nombre d'entre eux, un ensemble de références à la possibilité d'une transformation révolutionnaire de la société. De la même façon, la domination exercée par les communistes français sur le mouvement ouvrier n'est pas le simple produit du hasard d'une conjoncture. En fait, au cours des années 1934-1938, le PCF a gagné la majorité dans les entreprises, les quartiers, le mouvement syndical à l'occasion de la grève générale de juin 1936 et de ses prolongements. C'est ainsi qu'en dépit du confusionnisme de leur pratique, les communistes français ont joué un rôle historique: ils ont contribué à donner au mouvement ouvrier une organisation de masse, qui n'avait jamais existé jusque-là en France. À la différence des pays européens où, dès la fin du XIXe siècle, la socialedémocratie avait construit des réseaux d'organisations, véritable contre-société, qui avaient façonné l'existence publique de la classe ouvrière. Pour résumer, le PCF vit, dans ces années de guerre froide dans une situation de contradiction structurelle. Il incarne avant tout une bureaucratie dont l'origine double - stalinisme et mouvement ouvrier intégré - dresse un obstacle quasi-absolu à tout fonctionnement démocratique ${ }^{7}$. Il est donc totalement illusoire de croire qu'une crise de société sera demain ou après-demain susceptible de dégager une aile révolutionnaire dans l'organisation: le contexte dans lequel s'inscrirait une éventuelle réorientation du parti serait marqué par la pesanteur des liens attachant un parti, bureaucratisé à l'extrême dans son fonctionnement national, à un système contre-révolutionnaire international. Cependant, ce constat réaliste n'implique nullement que le mouvement communiste français est incapable de réagir à une conjoncture de crise politique et de développement des luttes. Bien au contraire, ses liaisons internationales avec le « socialisme réellement existant » le conduisent à prendre pratiquement en compte les aspects les plus globaux de la stratégie politique. De même, 
sa position dirigeante dans le mouvement ouvrier français le fait participer obligatoirement aux réflexions sur les perspectives politiques qu'ouvrent grèves et manifestation de masse. Il faut seulement comprendre que l'évolution d'un parti comme le PCF ne peut être que lente, dispersée et ne peut donc aboutir à des conclusions positives (du point de vue révolutionnaire) que si les modalités d'élaboration d'une politique nouvelle tiennent compte des contradictions qui rigidifient la pensée première des militants.

\section{La fin de la nuit}

11 Il fallut attendre jusqu'à 1956 pour que se révèlent publiquement les contradictions du «monde communiste». En France, deux séries de faits vinrent perturber le rapport qu'entretenaient les militants avec la situation française. En 1956, c'est le moment où la guerre coloniale que mènent les Français en Algérie entre dans une nouvelle phase. Sous la conduite de Guy Mollet, dirigeant de la SFIO, les moyens de la répression de l'autre côté de la Méditerranée vont atteindre des proportions scandaleuses : le "contingent » (les jeunes hommes qui accomplissent leur service militaire) est envoyé faire la guerre sur place; en même temps, la répression contre les Algériens prend les dimensions d'un crime contre l'humanité (l'emploi de la torture se généralise). Pour mener à bien cette politique, Guy Mollet a besoin du soutien de l'opinion publique ; il demande au Parlement l’octroi de "pouvoirs spéciaux ». Et les députés communistes les votent ! Cette décision choque profondément nombre de militants, formés dans la tradition anticolonialiste du mouvement communiste ${ }^{8}$. Le mécontentement gagna de nombreuses cellules, en particulier celles qui avaient un contact militant avec des Algériens résidant en France. Aux critiques formulées par la base, la direction du PCF trouva à répondre que l'existence d'un gouvernement de gauche ouvrait des perspectives telles que la guerre d'Algérie était réduite en importance. «Il ne faut pas sacrifier le tout (le gouvernement Mollet) à la partie (la guerre d'Algérie)»: telle était la formule communiste employée par les responsables du parti. On peut imaginer à quel point cette inintelligence formalisée créa dans le parti un malaise, même si celui-ci ne se traduisait pas par des critiques politiques ouvertement prononcées. En Union Soviétique se déroulait, au début de la même année 1956, le XXe congrès du PCUS. Dans son rapport, prononcé à huis clos mais largement diffusé après-coup, Khrouchtchev amorça une critique du stalinisme, vu sous l'angle du « culte de la personnalité ». En d'autres termes, pour la première fois depuis sa naissance, le système bureaucratique était - partiellement - dénoncé par ceux-là mêmes qui en étaient les protagonistes. Ce seul fait était à l'origine d'un trouble dans les partis communistes qui voyaient mises en doute les certitudes nées de la confiance absolue dans les régimes « communistes ». En France, le malaise fut encore plus grand dans la mesure où la direction du parti feignit de ne pas croire à l'authenticité du «rapport Khrouchtchev ». Peu de temps après - à l'automne 1956 - survint ce que l'on est en droit d'appeler la "révolution hongroise", vite étouffée dans le sang par l'intervention militaire soviétique. La confusion atteint un maximum dans le PCF. On y répandit en effet la fausse information selon laquelle la présence des chars soviétiques à Budapest était justifiée par la menace d'un coup d'Etat fasciste ${ }^{9}$. Et, en définitive, c'est surtout dans les milieux intellectuels, qui avaient accès aux informations suffisantes, que l'affaire hongroise entraîna une crise sérieuse. Mais, dans l'ensemble du parti, la jonction des doutes et des mécontentements nés de l'Algérie et des pays «socialistes " contribua à 
gripper le moteur du bel ensemble bureaucratique qu'avait été jusque-là le PCF. Il devenait dès lors possible d'envisager une opération militante tendant à organiser un courant politique au sein même du parti.

\section{Tribune de discussion} participé à la campagne des trotskistes en défense de la Yougoslavie, victime d'une agression politique de la part du camp stalinien. Il avait par la suite, pour des raisons personnelles, pris ses distances avec l'organisation trotskiste, sans pour autant cesser de militer (en particulier avec le "groupe Hispano »). Il n'était pas membre du PCF, mais il entretenait des liens assez étroits avec plusieurs militants de la «cellule de philosophie " de la Sorbonne. Parmi eux, Lucien Sebag, Michel Cartry, Anne Giannini. Le petit groupe ainsi constitué avait vécu intensément les événements de l'année 1956, du XXe congrès au vote des pouvoirs spéciaux. Vigoureusement opposés à la politique de la direction du PCF, les «philosophes» étaient en quête d'une explication théorique globale de la dégénérescence du régime né de la Révolution d'Octobre. Ils entendaient, en même temps, porter leurs critiques au-delà des limites de leur cellule. Ce fut l'objectif principal de leurs discussions avec Félix Guattari. Celui-ci leur fit une proposition: éditer un bulletin dont l'objectif serait de rendre publiques les critiques à l'encontre de la ligne du PCF et d'assurer ainsi un débat démocratique, le plus large possible, comme il eût été normal qu'il se déroulât dans le parti. Félix insista beaucoup sur la nécessité d'éviter tout dogmatisme: la publication projetée ne se présenterait pas comme porteuse d'un programme pré-élaboré; elle viserait plutôt à être un instrument de réflexion qui fournirait à ses lecteurs la possibilité de parvenir, par le biais de la confrontation, à une élaboration des principales réponses aux questions que posait l'actualité.

Le contact fut pris avec Pierre Frank, qui promit le soutien de son group $\mathrm{e}^{10}$. La direction me désigna pour exercer la responsabilité de la publication, à partir de son deuxième numéro. C'est ainsi que naquit la Tribune de Discussion. Avec le recul des années, il est possible de dresser un bilan de l'opération. Publié avec une régularité relative, le bulletin ronéotypé (à l'exception de son dernier numéro, imprimé) eut une diffusion limitée (le fichier se composait pour l'essentiel des adresses recueillies autour des militants). Malgré ces limitations graves, l'initiative rencontra un écho certain parmi les intellectuels communistes : si l'on excepte le bulletin Unir pour le socialisme, aux origines plus que douteuses, c'était la première fois depuis trente ans que paraissait un organe d'opposition au sein du PCF. La publication de la Tribune de Discussion fut aussi l'occasion de coordonner le travail des militants «entristes». Un collectif d'animation fut créé. Y participèrent des communistes récemment venus à l'opposition. Et aussi des intellectuels connus, tels Henri Lefebvre et François Châtelet.

Bref, en dépit du manque de moyens et d'une préparation quasi inexistante, l'opération Tribune fut loin d'être un échec. Qu'elle ait trouvé écho principalement dans l'intelligentsia s'explique aisément : la crise du PCF n'en était qu'à ses débuts, les questions politiques n'apparaissaient que partiellement; rien de surprenant à ce que la contestation fut d'abord l'apanage de professionnels de la pensée. 
15 En tout état de cause, l'initiative prise alors pouvait n'être qu'une étape préparatoire à un combat de plus vaste envergure. La méthode adoptée - élaboration d'une orientation à partir des débats liés à l'expérience - correspondait à la vraie « nature » du PCF. Comme on l'a rappelé précédemment, le malaise interne du PCF, un moment dévié par la préparation du Congrès du parti, reprit avec ampleur à l'automne sous l'influence des " événements » de Hongrie. Là encore, les intellectuels communistes eurent un rôle décisif. Leur révolte ne se limita pas aux problèmes posés par leur activité professionnelle. Elle eut immédiatement une dimension politique. Une fois de plus, il fut clairement démontré que lorsque, pour des raisons diverses, les militants ouvriers ne sont pas en mesure de prendre une part active à l'élaboration d'une politique opposée à celle des dirigeants bureaucratiques, intellectuels et étudiants peuvent occuper une position d'avant-garde, quand bien même leur coupure avec les luttes populaires limite leur expression.

En 1956, les intellectuels du PCF contribuèrent, dans de nombreuses circonstances, à accélérer les manifestations de la crise du parti. Ils généralisèrent les critiques qui, depuis le XXe congrès du PCUS, avaient commencé à circuler. Beaucoup d'exemples, jusque-là tus, des fautes de la direction furent évoqués publiquement. On enregistra la démission de militants connus, parfois de longue date au parti. Mais le plus significatif fut qu'autour d'un noyau composé de cadres du parti, pour la plupart présents dans l'organisation depuis les années 1930, se constitua ce que le vocabulaire politique de l'époque désignait comme une fraction - autrement dit un groupe structuré, organisant clandestinement des interventions à l'intérieur du PCF pour promouvoir une ligne opposée à celle de la direction. Victor Leduc, Jean-Pierre Vernant, Anatole Kopp et bien d'autres encore furent des artisans de la constitution de ce pôle politique. Tous avaient exercé des responsabilités, non seulement dans le milieu correspondant à leur spécialité professionnelle, mais aussi dans la presse du PCF et dans les commissions de travail de la direction $d u$ Parti $^{11}$. Les initiatives $d u$ rassemblement ainsi constitué furent immédiatement confrontées au problème de la diffusion de leurs idées auprès des militants communistes. Ils pensèrent un moment se rallier à Tribune de Discussion ${ }^{12}$. Mais le passage à l'acte ne put se réaliser pour deux raisons principales. Tout d'abord, le noyau qui assurait la publication du bulletin était composé de militants jeunes, inconnus dans le PCF en dehors du niveau local où se déroulait leur action. Aussi bien la question se posait de savoir qui était à l'origine de la publication ${ }^{13}$. Mais surtout, une sérieuse divergence politique entre les deux groupes apparut très vite, à propos de la Hongrie. On se souvient que le mouvement populaire anti-stalinien qui avait atteint une dimension de masse avec la constitution de conseils ouvriers dans les entreprises fut écrasé par une seconde intervention soviétique (4 novembre 1956) qui se solda par plus de 20000 morts et par l'élimination de toutes les institutions démocratiquement formées à Budapest et dans l'ensemble du pays. La Tribune de Discussion condamna sans ambages cette action contrerévolutionnaire. Ce refus signifiait un rejet sans phrase du régime stalinien dont la vraie nature apparaissait clairement au gré de son rejet des initiatives politiques nées de la base. Conclusion trop radicale pour les militants communistes formés à l'école du " marxisme-léninisme » bureaucratique et habitués à n'envisager l'avenir qu'en fonction du rôle «dirigeant» de l'Union Soviétique. Le groupe constitué autour de Leduc se prononça donc en faveur de la seconde intervention de l'armée rouge, en arguant, à la suite du PC chinois, des risques politiques (accentuation de la crise du bloc soviétique, remise en cause de la « dictature du prolétariat » incarné par le régime hongrois, etc.) 

publication. L'Étincelle fut donc tirée à plusieurs milliers d'exemplaires. C'était un journal dont les perspectives étaient nettement exposées dans un mot d'ordre qui figurait sous le titre du journal: "Pour le redressement révolutionnaire du Parti Communiste». L'ensemble des articles et des correspondances publiés avait, dans le contexte de l'époque, une qualité qui tranchait avec le contenu et la forme de la presse du PCF de l'époque. Il nous apparut donc nécessaire de recherches les moyens d'un travail en commun avec les militants regroupés autour de l'Étincelle.

19 Des contacts furent pris assez rapidement. Des délégations se rencontrèrent et menèrent des discussions de fond. On arriva à un accord de fusion : l'Étincelle paraittrait désormais avec le sous-titre Tribune de Discussion. Des groupes locaux seraient organisés, non seulement pour aider à la diffusion du bulletin, mais pour assurer la défense des positions critiques communes. Nous insistâmes sur deux demandes : la constitution d'un exécutif où les militants des deux anciens bulletins seraient représentés sur un mode paritaire ; la rédaction d'une " plate-forme » qui exprimerait les bases de notre accord politique sur les problèmes du mouvement communiste international aussi bien que sur l'analyse de la situation française et les conclusions pratiques que l'on pouvait en tirer. Ces propositions furent acceptées sans difficulté.

20 Par contre, elles suscitèrent des remous au sein de la direction du groupe trotskiste. Lorsque je les exposai, elles se heurtèrent aux critiques de Pierre Frank qui déclara avec assurance que c'était une erreur que de proposer la rédaction d'une plate-forme politique car un tel document risquait d'empêcher l'évolution ultérieure des militants rassemblés autour de l'Étincelle; il convenait de laisser la crise du stalinisme faire surgir de nouvelles interrogations qui se traduiraient par de nouvelles prises de position, plus proches des nôtres. Je répondis, soutenu par des camarades qui partageaient mon expérience, que la dite « plate-forme » n'incarnait qu'un moment de la lutte commune menée au sein du PCF et qu'elle était un moyen d'inciter des militants, pour une part, déformés par l'école stalinienne, à réfléchir par eux-mêmes aux conclusions fondamentales qui pouvaient être tirées de chaque époque de la stratégie. Une telle démarche était la condition même de la construction d'une organisation révolutionnaire, car il était évident que le développement de la crise du monde bureaucratique serait marqué par le surgissement de problèmes inédits sans pourtant que ceux-ci aboutissent automatiquement à des prises de positions satisfaisantes. C'est alors que Jacques Privas fit une de ces interventions dont il avait le secret. Il nous expliqua sans rire que c'était une faute que d'avoir demandé - et obtenu - la parité dans l'exécutif de la nouvelle Étincelle : il fallait laisser la majorité aux militants venus du PCF car, comme l'avaient exprimé les thèses des congrès de la IVe Internationale, des pans entiers des grandes organisations s'orienteraient vers une politique révolutionnaire, notre tâche étant de les accompagner.

21 Je cite cette discussion car elle me semble révélatrice d'une myopie du trotskisme dans le domaine de la réflexion sur la construction d'un parti. Les positions dans ce domaine sont une référence permanente - et mécanique - aux mouvements sociaux d'ampleur historique qui automatiquement produisent les évolutions souhaitables. Ce déterminisme largement artificiel est incapable d'aider à concevoir que la construction d'une organisation passe d'abord par l'apprentissage de l'autonomie individuelle et collective.

Revenons à l'Étincelle, en cette année 1957 où la fusion des deux groupes s'est réalisée. Les conséquences du XXe congrès se font toujours ressentir, mais sur un rythme ralenti. Le travail en commun progresse sans hâte. En Union Soviétique, Khrouchtchev renforce sa 
position avec l'élimination du « groupe anti-parti ». Où allons-nous? est la question que se posent les militants critiques. C'est dans ce contexte que va se dérouler une crise de l'opposition communiste. Une ancienne dirigeante pabliste, Michèle Mestre, nous dénonce publiquement comme trotskistes. On devine les réactions des uns et des autres. Les animateurs de l'Étincelle qui subissent par ailleurs des pressions répétées de la direction du PCF ne peuvent continuer à travailler avec des gens qui portent cette étiquette infamante. Mais il faut trouver un prétexte politique à la rupture. C'est le choix du motif qui est intéressant, par ce qu'il révèle du mode de penser de militants qui ont subi la (dé)formation stalinienne. Pour satisfaire la direction du parti, on va dissoudre officiellement le groupe rassemblé autour du bulletin fractionnel; pour répondre aux interrogations des militants, on va inventer une justification politique à cette dissolution (et, dans l'affaire, on se débarrassera des trotskistes). C'est ainsi que, sans rire lui non plus, Victor Leduc explique un jour que l'élimination des Malenkov, Molotov et autres membres du « groupe anti-parti » implique que, désormais, la déstalinisation se produira automatiquement - ce qui rend inutile, voire dangereux, tout travail fractionnel.

A suivre dans notre prochain numéro

\section{NOTES}

1. L'honnêteté et la fidélité à Freud me poussent à signaler l'influence de mon frère aîné, qui fut un temps lecteur de la Vérité, hebdomadaire du PCI.

2. Il va de soi que la pensée de Trotsky, à la veille de la guerre comme lors des années précédentes, est infiniment plus riche que ne peut l'indiquer un bref résumé de ses propositions d'action. Pourtant, l'énoncé de directives d'action, justifiées par une analyse prospective des grands rapports de forces dans le monde, révèle des erreurs de méthode aux conséquences redoutables.

3. L'avenir allait montrer que le déclin et la chute des États bureaucratiques, long processus historique, avaient eu leur point de départ dans l'« affaire yougoslave ».

4. Il est évident que les critiques formulées ici à l'encontre d'un certain «matérialisme historique " - réduit en fait à un "matérialisme sans histoire »- ont pour cible principale les théoriciens de la Deuxième Internationale qui, ne l'oublions pas, ont largement contribué à la formation de Lénine. Mais la question reste ouverte des faiblesses dans les démonstrations de Marx et Engels eux-mêmes, faiblesses qui ont ouvert la voie à moult errements. Ce n'est pas ici le lieu d'un tel débat. Toutefois, il m'a semblé utile d'indiquer au passage que le respect des traditions révolutionnaires, indéniable chez les trotskistes, pouvait signifier aussi le maintien d'une lourdeur nuisible, sans l'application de la méthode à une conjecture inédite.

5. Notons seulement que la rupture au sein de la IVe Internationale, si elle fut facilitée par la nature des affrontements politiques, fut grandement accélérée par les méthodes expéditives de la direction, Michel Pablo principalement.

6. On les appelle les « pablistes ", du nom de Michel Raptis, dit Pablo.

7. La suite des événements le montrera : il faudra plusieurs décennies pour que le parti formule une vision critique du stalinisme et de son propre passé. Et encore, les condamnations prononcées alors seront le fait des seuls dirigeants, sans qu'un débat général soit ouvert aux 
militants. En fait, c'est seulement ces dernières années qu'un peu de liberté de discussion fut introduit dans le PCF.

8. Tradition qu'a fait revivre, peu de temps auparavant, la lutte contre la guerre du Vietnam ; les communistes n'hésitèrent pas à utiliser des moyens de combat hors légalité.

9. À Paris, des manifestants, fortement influencés par la droite, s'attaquèrent aux locaux du PCF et de l'Humanité. Cette agression contribua au désarroi des militants communistes dont beaucoup tenaient à identifier les révoltés de Hongrie avec les trublions de Paris.

10. Les militants de la "cellule philo" rejoignirent le mouvement trotskiste, la plupart, tel Lucien Sebag, pour quelque temps seulement.

11. Victor Leduc, qui fut au centre de cette initiative, avait adhéré au PCF lorsqu'il était étudiant, dans les années 1930. Il joua un rôle important dans la Résistance - à Toulouse notamment. Après guerre, il fut successivement éditorialiste à l'hebdomadaire Action, puis responsable de la « commission idéologique » du parti (travail intellectuel).

12. Selon ce que m'a affirmé à l'époque Yves Cachin, un des initiateurs du groupe.

13. V. Leduc et ses proches se demandèrent sérieusement si le PC Yougoslave n'était pas l'initiateur - et le soutien financier - de Tribune de Discussion. 